\title{
Mortimer Sellers*
}

Traducción de

\author{
Juan David Almeyda Sarmiento** \\ Universidad Industrial de Santander (Bucaramanga, Colombia) \\ juanalmeyda96@gmail.com
}

\section{Nicolás Maquiavelo: padre del constitucionalismo moderno ${ }^{* * *}$}

\author{
Niccolò Machiavelli: Father \\ of Modern Constitutionalism
}

* Mortimer Newlin Stead Sellers es Profesor Regente de la Universidad System de Maryland,
Director del Centro para el Derecho Internacional y Comparado de la Universidad de Baltimore,
Profesor de la Facultad de Derecho de esta misma Universidad y Presidente de la Asociación
Internacional para la Filosofía del Derecho y la Filosofía Social (IVR). ORCID: https://orcid.
org/0000-0001-6648-6937
**Egresado y profesor de cátedra del programa de filosofía de la Universidad Industrial de Santander.
Revisado por: Andrés Botero Bernal (Profesor titular de la Escuela de Filosofía de la Universidad
Industrial de Santander).
*** Este artículo es una traducción del siguiente texto: (Sellers, 2015: 216-225). 



\title{
Resumen
}

Nicolás Maquiavelo es el padre del constitucionalismo moderno. El constitucionalismo comenzó de nuevo en el mundo moderno con el estudio de las antiguas repúblicas y fue Maquiavelo quien inauguró esta ciencia política revivida. Quinientos años después de la escritura de Il principe y los Discorsi, aún estamos trabajando en las implicaciones de aplicar la razón a las estructuras del derecho y del gobierno en búsqueda de la justicia y del bien común. El constitucionalismo moderno y el republicanismo antiguo comparten tres creencias centrales: primero, que el gobierno debe servir a la justicia y al bien común; segundo, que el gobierno debería hacerlo a través de leyes conocidas y estables; tercero, que estas serán mejor aseguradas a través de los pesos y contrapesos de una constitución bien diseñada. Maquiavelo tomó las teorías y experiencias de la Roma republicana y las aplicó a su propia época. Esta aplicación de la razón al diseño constitucional transformó la política de la modernidad emergente y reconfiguró el gobierno en todo el mundo.

Palabras claves: Nicolás Maquiavelo, constitucionalismo, filosofía del derecho, historia del derecho.

\begin{abstract}
Niccolò Machiavelli is the father of modern constitutionalism. Constitutionalism began anew in the modern world with the study of the ancient republics and it was Machiavelli who inaugurated this revived science of politics. Five hundred years after the composition of $I l$ principe and the Discorsi we are still working out the implications of applying reason to the structures of law and government in pursuit of justice and the common good. Modern constitutionalism and ancient republicanism share three central beliefs: first, that government should serve justice and the common good; second, that government should do so through known and stable laws; third, that these will best be secured through the checks and balances of a well-designed constitution. Machiavelli took the theories and experiences of republican Rome and applied them to his own era. This application of reason to constitutional design transformed the politics of emergent modernity and reconfigured government throughout the world.
\end{abstract}

Keywords: Niccolò Machiavelli, Constitutionalism, Philosophy of law, Law history. 



\section{Introducción}

Hace quinientos años, Nicolás Maquiavelo completó Il Príncipe, su famoso manual para príncipes (Maquiavelo, 2001) ${ }^{1}$. Algunos consideran que este evento fue el comienzo de la ciencia política moderna, lo cual puede ser cierto. Pero el mayor aporte de Maquiavelo surge de su Discorsi sopra la prima deca di Tito Livio (Maquiavelo, 1987), que escribió aproximadamente al mismo tiempo. Menos discutidos hoy que Il principe, los Discorsi han tenido una influencia más profunda y duradera. Los Discorsi eran más inusuales, más revolucionarios y transformadores que Il principe cuando fueron escritos, y han dejado un legado más significativo. Mediante ellos Maquiavelo se convirtió en el padre del constitucionalismo moderno. Él revivió el legado de la antigua teoría republicana, transformó la forma de la política en la modernidad emergente y reconfiguró el gobierno en el mundo moderno. Maquiavelo cambió la dirección del discurso político y todos sus sucesores más importantes han reconocido la prioridad de sus ideas.

La posición de Maquiavelo como el padre del constitucionalismo moderno tiene tres principales aspectos: paternidad, modernidad y constitucionalismo, cada uno de los cuales requiere definición y merece un examen más detenido. "Padre" aquí quiere decir que Maquiavelo fue el creador y el cuidador de la tradición constitucionalista (Sellers, 1998). "Modernidad" hace referencia al proyecto de reemplazar la autoridad con la razón, que inauguró el mundo moderno (Sellers, 2009). El "constitucionalismo" es la lucha por construir la mejor forma de gobierno para cualquier sociedad, en el caso de Maquiavelo, principalmente para Florencia (Sellers, 2014). El enfoque central de esta discusión será el constitucionalismo y la innovación de Maquiavelo, pero la modernidad también juega un papel importante. El constitucionalismo moderno comienza en cualquier sociedad cuando los gobernantes aplican su razón a la estructura del Estado para asegurar un mejor gobierno y mejores leyes. Maquiavelo revivió el proyecto de la razón política en Florencia por medio de un retorno a la tradición constitucional de la Roma republicana.

Esto plantea la relación entre "republicanismo" y "constitucionalismo", y, más específicamente, la medida en que la antigua concepción del republicanismo

$1 \mathrm{~N}$. del T: el artículo cita las versiones originales de Il principe y los Discorsi, pero para la traducción se utilizaron versiones oficiales ya publicadas en español para facilitar la compresión de los libros de Maquiavelo. 
condujo a teorías contemporáneas del gobierno constitucional. Existe una conexión directa entre ambas. Las teorías contemporáneas del gobierno constitucional surgen casi enteramente de antiguas ideas republicanas. Ellas son una extensión del mismo esfuerzo. El constitucionalismo moderno es simplemente un renacimiento y continuación de la antigua tradición republicana bajo otro nombre. Nicolás Maquiavelo fue el más importante autor de esta resurrección y, por lo tanto, es el padre del constitucionalismo moderno. Maquiavelo redescubrió el constitucionalismo y la ciencia de la política republicana después de un milenio de tiranía y opresión.

\section{Constitucionalismo}

¿Cúal es, entonces, el proyecto constitucionalista que Maquiavelo revivió para el mundo moderno? El constitucionalismo es una propuesta que propende al diseño de una estructura de gobierno que sirva a la justicia y al buen gobierno. Maquiavelo hizo esto especialmente en sus Discorsi sopra la prima deca di Tito Livio (Livio, 1974). Maquiavelo buscó sistemáticamente estudiar los ejemplos tanto de la antigüedad como de la política moderna para constituir una mejor república y perfeccionar la estructura del Estado. Afirmó como primer objetivo el beneficio común o "commune benefizio" de todos los sujetos dentro de la república (Maquiavelo, 1987, I. proemio. 1). Esto fue y es (como debería ser) ampliamente reconocido, en su tiempo como en el nuestro, como el propósito principal y la justificación de todo gobierno. Maquiavelo explicó que en la búsqueda de instituciones políticas dirigidas para el bien común él aplicaría a la política y a la constitución una técnica realmente bien conocida en otras artes y ciencias. Maquiavelo buscó emplear su propia razón al conocimiento y experiencia de los antiguos para aprender la mejor manera en que es posible construir repúblicas, tal como los escultores italianos contemporáneos copiaron antiguas estatuas, o como los arquitectos estudiaron a Vitrubio para crear obras de arte más bellas y mejor construidas.

Esta sería, entonces, la técnica constitucionalista. Mientras los abogados estudiaban el derecho civil romano, y los médicos estudiaban a Galeno, de la misma forma Maquiavelo se dispuso a diseñar lecciones prácticas a partir del estudio de la historia para recuperar la libertad y la virtud de los antiguos. Maquiavelo afirmó que este enfoque era una innovación e insistió en que estaba haciendo un progreso vital para la política italiana. Afirmó ser el primer político moderno en aplicar la razón a la historia para aprender las lecciones del buen 
gobierno. Esto, de hecho, era algo nuevo tanto para su época como para el mundo moderno. Como en todas las demás áreas del conocimiento, el estudio de la política había estado estancado, incluso en regresión, desde la caída de Roma. Para superar a los antiguos en cualquier campo, los modernos primero deben entenderlos. Maquiavelo convirtió a sus contemporáneos modernos al conocimiento y la experiencia de los antiguos, de modo que fuera el primer paso para aplicar la razón a la política, y a cualquier otro campo. Para avanzar en cualquier ciencia uno debe comenzar con la razón y la realidad, Maquiavelo encontró estas dos cosas en el mundo antiguo.

El constitucionalismo comparte con el antiguo republicanismo una teoría respecto del propósito del gobierno (servir a la justicia y al bien común), una visión del método para hacerlo (a través de leyes conocidas y estables) y una técnica para asegurar el éxito (a través de los pesos y contrapesos de una arquitectura constitucional bien diseñada). Maquiavelo tomó tanto las teorías como la experiencia de la Roma republicana y los aplicó a las dificultades políticas que enfrentaban en su propia época Florencia, Italia y Europa. ¿Cómo puede la organización política asegurar un gobierno para el bien común en un contexto lleno de violencia, avaricia, ambición, corrupción, superstición y poder fuera de lugar como lo era Europa cuando Maquiavelo escribió Il principe y sus Discorsi? Maquiavelo sugirió que los pesos y contrapesos entre los diferentes poderes en el sistema de gobierno podrían asegurar mejores resultados que el poder sin control en manos de cualquier individuo, facción o partido. Propuso la elección de funcionarios públicos y respeto al Estado de derecho. Y él apoyó estas propuestas citando la experiencia de la Roma republicana.

\section{Paternidad}

Maquiavelo pensó que estaba haciendo algo nuevo e importante al revivir la política republicana para el mundo moderno, pero para ser el padre metafórico de una tradición intelectual uno debe tener hijos metafóricos y estos deben reconocer la paternidad de uno a sus ideas. La evidencia decisiva de la influencia de Maquiavelo es el testimonio de sus sucesores, que está demostrado en la sinceridad de las frecuentes desaprobaciones de sus seguidores con él. Todos los grandes héroes del constitucionalismo moderno reconocen a Maquiavelo como su padre intelectual e inspiración, incluso cuando riñeron con su carácter y sus conclusiones en un intento por mejorar las ideas de él. 
La Revolución Inglesa, la Revolución Estadounidense, la Revolución Francesa y todo el ímpetu moderno por la reforma constitucional comenzó con los Discorsi de Maquiavelo. James Harrington en Inglaterra (Harrington, 1977), John Adams en Estados Unidos (Adams, 1787-1788), el barón de Montesquieu (Montesquieu, 1748) y Jean-Jacques Rousseau (Rousseau, 1762) en Francia, todos se basaron en los escritos de Maquiavelo, pero también en los antiguos autores en quienes él también se había fundado: Cicerón (Cicerón, 1913) (Cicerón, 1928), Polibio (Polibio, 1922), Salustio (Salustio, 1931), Tácito (Tácito, 1906) (Tácito, 1911), Plutarco (Plutarco, 1949-1971), Platón (Platón, 1902), Aristóteles (Aristóteles, 1957) y, por supuesto, Livio (Livio, 1919), como lo hizo en los Discorsi. Ellos se vieron a sí mismos como parte de la misma tradición que Maquiavelo había revivido con sus discusiones de Livio y de Roma.

El siguiente gran triunfo del constitucionalismo y el pensamiento constitucional después de la Florencia de Maquiavelo estuvo en la lucha inglesa contra los monarcas estuardos, culminando en la Revolución Gloriosa de 1688. James Harrington, quien propuso una nueva Constitución de libertad para Inglaterra en su Oceana, citó a Maquiavelo como "el único político" (es decir, el único estudiante de ciencia política) desde la antigüedad. Harrington elogió a Maquiavelo por recuperar lo que llamó "la prudencia antigua" y "el imperio de las leyes y no de los hombres" (Harrington, 1977: 8-10)². Cuando los estadounidenses buscaron establecer un gobierno constitucional un siglo después, ellos también recurrieron a Maquiavelo, sus fuentes y a aquellos que siguieron en su tradición [la de Maquiavelo]. John Adams, el principal teórico constitucional de la Revolución Estadounidense, elogió a Maquiavelo como "el primero que revivió la política antigua" para el mundo moderno. En esto también siguió a los filósofos franceses. Adams observó que Montesquieu "tomó prestado de Maquiavelo la mejor parte de su libro, sin reconocerle [a Maquiavelo] las citas", y que "Milton, Harrington y Sidney, estaban íntimamente familiarizados con los antiguos y con Maquiavelo" (Adams, 1787-1788, I.325) .

$2 \mathrm{~N}$. del T: todas las citas textuales, excepto aquellas de Maquiavelo, son traducción propias del traductor de este documento.

3 Cf. (Harrington, 1977) (Milton, 1991) (Sidney, 1698). 


\section{Modernidad}

La modernidad se traduce como el proyecto de reemplazar la autoridad con la razón. Esta actitud llegó a Europa en diferentes momentos de acuerdo con las distintas disciplinas entre los siglos XII y XVI, pero para el caso de la política y el constitucionalismo fue con los escritos de Maquiavelo mientras intentaba reformar el Estado florentino. Por supuesto, "modernidad" en su sentido más simple sencillamente indica lo que es más reciente, y esto puede causar confusión. Hubo un tiempo en que "moderno" significaba hablar de la era cristiana, en oposición a la "antigüedad" que la precedió. Solo más tarde la "modernidad" se asoció con la nueva era de renacimiento e ilustración (Sellers, 2009: 247). Maquiavelo y Harrington escribieron en oposición a la tiranía y la anarquía de su propia época, y recurrieron al ejemplo de Roma para encontrar el coraje de desafiar las instituciones existentes. El constitucionalismo republicano de Roma era la "antigua prudencia" que buscaban revivir. "La prudencia moderna" (en este vocabulario más reciente) seguía siendo la política sin ley de la Europa contemporánea: la política que Maquiavelo y sus sucesores esperaban reemplazar con tanto fervor.

El constitucionalismo moderno, en su núcleo, era simplemente un renacimiento de la antigua tradición republicana. James Harrington dividió toda la historia del gobierno en dos grandes épocas: la primera, "terminando con la libertad de Roma", la cual llamó "prudencia antigua" o gobierno "de jure", definido como "el arte por el cual una sociedad civil de hombres se instituye y preserva sobre la fundación del derecho e interés comunes". Harrington elogió a Livio y Aristóteles por abogar por la prudencia antigua y por avanzar en lo que él llamó el "imperio de las leyes y no de los hombres", es decir el "gobierno constitucional" o "constitucionalismo", en nuestro más moderno vocabulario. El otro tipo de gobierno comenzó, dijo Harrington, "con las armas de César" quien "extinguiendo la libertad" creó lo que en la época de Harrington todavía era llamado "prudencia moderna" o gobierno "de facto", que había "deformado toda la faz del mundo con esas características precarias en lo correspondiente a la forma de gobernar [...] donde algunos hombres, o algunos pocos hombres, someten a una ciudad o una nación, de acuerdo a su propio interés". Este es "el imperio de los hombres y no de las leyes", porque los gobernantes son capaces de hacer e interpretar las leyes solo para su propio beneficio (Harrington, 1977: 13).

El gobierno "de jure" propuesto por Harrington es lo que ahora llamaríamos "constitucionalismo", y atribuyó sus ideas sobre este punto, y sobre la "prudencia 
antigua", a Nicolás Maquiavelo. Harrington contrasta a Maquiavelo con Thomas Hobbes, el gran defensor de la "prudencia moderna" o gobierno de facto, donde los gobernantes no enfrentan restricciones constitucionales (Harrington, 1977: 13). Al hacerlo, Harrington reconoció a Maquiavelo como el padre del constitucionalismo moderno. Señala a Maquiavelo como el primer estudiante moderno de la sociedad política y del gobierno por el bien común. Su fuente principal para esto fueron los Discorsi sopra la prima deca di Tito Livio. Los Discorsi, tienen un enfoque hacia la antigüedad, sin embargo, en muchos sentidos son un documento mucho más moderno que Il principe, que trata más sobre los asuntos contemporáneos. Il principe, un breve manual de tácticas para príncipes, cuestionó un muy gastado modelo con franqueza y perspicacia notables, pero los Discorsi desafiaron la base misma del Estado. Los Discorsi consideran no solo cuál sería el marco ideal de gobierno en diversas culturas y circunstancias, sino que también traen a colación la pregunta más profunda y difícil respecto a cómo salir de la violenta y corrupta política de Italia y Europa, en tanto que este nuevo proyecto de Maquiavelo en realidad existía para pensar un mundo mejor donde tuvieran lugar la libertad, la justicia y el gobierno para el bien común.

\section{Constitucionalismo inglés}

Gran Bretaña produjo el primer gran triunfo del debate constitucional moderno cuando los partidarios de la Commonwealth emprendieron la Guerra Civil Inglesa (al menos en sus escritos) como una lucha por un gobierno constitucional mixto y equilibrado contra la tiranía y opresión. Aunque los ingleses sometieron a su rey a la ley y a la Constitución en 1649, nunca lograron un establecimiento sólido. Esto último comenzó la marcha hacia la libertad constitucional en Europa, que vio resultados reales y duraderos con la Revolución Gloriosa de 1688. James Harrington, el constitucionalista más sofisticado de esta época, colocó a Maquiavelo, junto a los autores antiguos, como "el único político de los últimos tiempos". Él vio a Maquiavelo como el primer defensor moderno del gobierno por medio de la razón, buscando asegurar el bien común con pesos y contrapesos en la constitución. Como Harrington lo explicó, los magistrados y el poder ejecutivo deben ser equilibrados por un senado independiente, que debe ser equilibrado, a su vez, por una asamblea popular más grande, de modo que se pueda crear una arquitectura tripartita del poder gubernamental, que sirva al bienestar del pueblo soberano. Harrington escribió que no podría ser "ninguna otra commonwealth sino esta, ya sea esta creada o natural, y esto 
no nos extraña puesto que Maquiavelo nos había mostrado que los antiguos mantuvieron esto como lo único bueno" (Harrington, 1977: 25).

Si bien fueron los propios reyes quienes precipitaron las revoluciones inglesas con su tiranía, Maquiavelo y las fuentes republicanas que reintrodujo en el discurso político proporcionaron modelos y un vocabulario para las nuevas instituciones constitucionales que reemplazarían el poder arbitrario de los reyes. Harrington reconoció su deuda con Maquiavelo. Honró a Maquiavelo como "el único recuperador de esta prudencia antigua" (Harrington, 1977: 30). Agradeció a Maquiavelo por revivir la visión de Aristóteles: "La multitud es más sabia y más constante que un príncipe.” (Maquiavelo, 1987, I. 58. 1). Maquiavelo sabía que el pueblo, si se les muestra la verdad, la seguirán, razón por la cual favoreció a una Roma más democrática sobre la cultura oligárquica que prevaleció en Venecia y en muchos otros Estados italianos.

Los republicanos ingleses y los partidarios de la Commonwealth encontraron sus reglas de gobierno en Maquiavelo y en sus modelos antiguos: Aristóteles, Livio, Cicerón y Polibio. El nuevo constitucionalismo siguió a Maquiavelo en la manera de explorar la historia romana y griega en búsqueda de evidencia empírica de los diferentes marcos de gobierno en la práctica. El énfasis principal no estaba tanto en las conclusiones de Maquiavelo como en su técnica. Harrington, Milton, Sidney y el resto compararon Roma con Esparta, Atenas y Venecia, en un intento por determinar qué instituciones serían más eficaces para garantizar la justicia y el bien común para todos los ciudadanos, y prevenir la tiranía de la monarquía en Inglaterra. Los experimentos italianos en diseño constitucional no habían sido un éxito sorprendente. Inglaterra ofreció el primer ejemplo de éxito en lo correspondiente a un gobierno constitucional en el mundo moderno. Los pesos y contrapesos del constitucionalismo inglés, su ideología sobre el Estado de derecho, las doctrinas de soberanía popular y el gobierno mixto que abrazaron tuvieron todos sus orígenes en Maquiavelo y en la Roma republicana.

\section{Constitucionalismo americano}

El siguiente gran éxito constitucional después de Gran Bretaña fue en América, comenzando con las constituciones estatales después de 1776 y luego con la Constitución Federal de 1787, hecha realmente en 1790. Aquí también, como en Gran Bretaña, Maquiavelo jugó un papel importante, junto con sus comentaristas ingleses y franceses, además de los antiguos modelos republicanos que él mismo había leído y discutido. Los Thoughts on government de John Adams (Adams, 
2000), que inspiraron las constituciones estatales, y su Defense of the constitutions of government of the United States of America (Adams, 1787-1788), que formó a la Constitución Federal, ambos siguieron el modelo maquiavélico de soberanía popular, gobierno mixto y Estado de derecho, como lo hizo la Constitución de Massachusetts en 1780, de la cual Adams fue principal diseñador. John Adams elogió a Maquiavelo por su realismo y por darse cuenta de que "quienquiera que funde un Estado, y promulgara leyes apropiadas para su gobierno, debe suponer que todos los hombres son malos por naturaleza, y que no dejarán de mostrar esa depravación natural del corazón cada que tengan una oportunidad". Adams señaló que Montesquieu también sabía esto y "la experiencia constante nos muestra que cada hombre investido de poder es apto para abusar de él: este tipo de desenfreno continúa hasta que encuentra algo que limita a dicho hombre". Entonces "para evitar el abuso de poder, es necesario que, por la disposición misma de las cosas, el poder sea un control para el poder mismo" (Adams, 17871788, I. 131). Esta es la esencia del constitucionalismo y la lección principal que Montesquieu, Adams, y los estadounidenses tomaron de Maquiavelo y de sus modelos: Cicerón, Livio, Polibio y demás.

Adams citó a Maquiavelo extensamente para corroborar la observación de que "los hombres nunca son buenos sino por necesidad: por el contrario, cuando el bien y el mal quedan a su elección, no dejarán de arrojar todo al desorden y la confusión". Por lo tanto, aunque "el hambre y la pobreza pueden hacer que los hombres sean laboriosos [.. .] solo las leyes pueden hacerlos buenos" (Adams, 1787-1788, I. 133-134). Este autor dedicó un capitulo entero de su Defense a la concepción de "gobierno mixto" propuesta por Maquiavelo, la cual dividió los poderes gubernamentales de tal manera que cada rama controlaría las demás. Maquiavelo alabó a Lycurgo por haber hecho esto de manera eficaz en Esparta y criticó a Solón por no hacerlo en Atenas. Pero, sobre todo, Maquiavelo sostuvo a la Roma republicana como el modelo constitucional ideal y Adams repitió su observación. Roma alcanzó "el más alto grado de perfección que cualquier comunidad es capaz de alcanzar" (Adams, 1787-1788, I.147).

\section{Republicanismo}

La ideología del constitucionalismo todavía no tenía vigencia bajo ese nombre cuando Harrington, Sidney, Montesquieu y Adams escribieron sus modelos para un gobierno constitucional, pues ellos hablaron de "repúblicas", y el republicanismo fue el proyecto que ellos, siguiendo a Maquiavelo, tenían 
esperanza de revivir. El "republicanismo" en su forma más simple no es más que un gobierno para el bien común, pero los republicanos, comenzando con Aristóteles y Cicerón, siempre habían entendido que no puede haber república sin el imperio de la ley, y no puede haber imperio de la ley sin la "forma republicana de gobierno", con todo lo que ello conlleva. La "gran pregunta", como dijo Adams, era:

qué combinación de poderes en la sociedad, o qué forma de gobierno, permitirá la formación de leyes buenas y equitativas, una ejecución imparcial y una fiel interpretación de ellas, de modo que los ciudadanos puedan disfrutar constantemente del beneficio de estas y se asegure su continuidad (Adams, 1787-1788, I.128).

La mayor virtud de Maquiavelo, a los ojos de los primeros constitucionalistas, fue que revivió esta pregunta y la puso sobre una base científica.

"Las artes y las ciencias en general", escribió John Adams en 1787, "han tenido un curso regular de mejora progresiva" en los últimos años, de modo que "los inventos en artes mecánicas, los descubrimientos de la filosofía natural, la navegación y el comercio" y "el avance de la civilización y la humanidad" han "ocasionado cambios en la condición del mundo y el carácter humano que habrían asombrado las naciones más refinadas de la antigüedad". Adams observó que "los pesos y contrapesos de los gobiernos republicanos han sido adoptados en cierto grado por las cortes de príncipes”. Adams, además, se percató de que "el conocimiento de los principios y de la construcción de gobiernos libres", conocimiento en el que "la felicidad de la vida, e incluso el mayor progreso en la educación y la sociedad en la sabiduría y la virtud, son profundamente ventajosas", ha permanecido "completamente detenido durante dos o tres mil años" (Adams, 1787-1788, I.i). La gran virtud de Maquiavelo a ojos de Harrington, Adams, Montesquieu y los demás, fue que revivió la ciencia del "gobierno libre", el gobierno de las leyes y el gobierno para el bien común. Estos autores leyeron y citaron a Maquiavelo porque él entendió y obró desde su comprensión que la construcción de constituciones y leyes es una ciencia accesible a la razón, como todas los demás.

John Adams y los otros fundadores del constitucionalismo moderno entendieron que el gobierno es una ciencia como todas las demás, solo que mucho más importante. De la misma forma que los arquitectos consultan a Vitruvio antes de construir, deben los gobernantes (dijo Adams) consultar a 
Maquiavelo, y también a Cicerón, Livio y Polibio, como el mismo autor florentino lo hizo. Los nuevos republicanos no copiaron servilmente o solo repitieron las prescripciones de Maquiavelo para el gobierno republicano, aunque a menudo sí lo hicieron, sino que lo involucraron a él y a los demás en un diálogo, basado en la razón y la experiencia, para desafiar y mejorar sus hallazgos. Las ideas constitucionalistas modernas surgieron del republicanismo antiguo y Maquiavelo fue el que más hizo para revivir esta tradición de investigación constitucional en Europa, o al menos se le dio crédito por haberlo hecho por parte de aquellos que hicieron más para construir sobre las teorías de Maquiavelo e incluso, a veces, implementarlas en la práctica.

\section{El constitucionalismo de Maquiavelo}

Habiendo examinado la profundidad de su influencia, deberíamos considerar brevemente qué puntos principales de Maquiavelo en realidad estaban relacionados con la política y la constitución ideal, y cuál de estos puntos causó la mayor impresión en sus lectores y sobre el surgimiento del pensamiento constitucional. Primero, en los Discorsi, Maquiavelo se dispuso a estudiar el ejemplo de la antigüedad y, sobre todo, de Roma, como se puede apreciar con Libio (1974). Segundo, su propósito al hacerlo era diseñar lecciones practicas de la experiencia de los romanos. Maquiavelo observó que los Estados deben tener poder si quieren sobrevivir, pero si quieren ser libres y tener justicia, ellos deben hacer leyes para el bien común, lo que requiere una mezcla constitucional y un equilibrio de los aspectos aristocráticos, monárquicos y democráticos del gobierno para crear una "republica perfetta", como la que existió en Roma 212 (Maquiavelo, 1987, I.2.30).

Maquiavelo identificó el secreto de la libertad y el poder de Roma en el equilibrio entre la plebe y el senado. Lo que hizo a la república libre y poderosa ("libera e potente") fue el conflicto constante entre los nobles y el pueblo (Maquiavelo, 1987, I.3.1). Maquiavelo favoreció la soberanía suprema del pueblo por dos razones. Primero, porque su libertad los hace dispuestos a luchar contra los enemigos de la república; y segundo, porque Maquiavelo creyó, siguiendo Cicerón, que el pueblo colectivamente juzga bien cuando los gobernantes ponen la verdad ante ellos. Por lo tanto, la libertad es, en última instancia, más segura en manos del pueblo y no en la de los "grandes hombres" ("grandi"), que compiten por el poder entre ellos. 
La parte más triste del ejercicio de Maquiavelo fue su convicción castigada de que será imposible crear un gobierno republicano en los Estados que han caído en la corrupción. La única ruta para salir de este callejón sin salida sería, quizás, encontrar un buen hombre dispuesto a usar malos métodos para lograr la reforma necesaria. Sin embargo, esto nunca puede suceder cuando la gente es tan corrupta como Maquiavelo creía que eran en Italia, Francia y España. Maquiavelo condenó a Italia como corrupta sobre todas las demás tierras y la fuente de una propagación de la corrupción que degradó al mundo entero, así que Florencia, por ejemplo, en toda su historia, nunca había sido realmente una república, porque era muy deshonesta. Maquiavelo percibió y, tristemente, registró que Florencia nunca había disfrutado de un buen gobierno porque Florencia nunca tuvo instituciones diseñadas para asegurar el bien común del pueblo, que es la esencia del gobierno republicano (Maquiavelo, 1987, I.18). El gobierno republicano es el gobierno que sirve a la res publica, y no algún interés privado o de alguna facción.

\section{Ciencia política}

Maquiavelo entendió que "todas las ciudades y todos los pueblos tienen los mismos deseos y las mismas pasiones, y así ha sido siempre" (Maquiavelo, 1987, I.39.1). Esto hace posible la ciencia política. Y sin embargo, Maquiavelo también vio, o creyó que vio, que donde no existe igualdad entre los ciudadanos, la gente se corromperá y será imposible establecer una república. Esta fue la situación en Italia. No es de extrañar, entonces, que Maquiavelo se recluyera en su estudio, como le dijo a su amigo Francesco Vettori, se pusiera su túnica curial, y entrara en diálogo con estos hombres antiguos, de modo que pudieran nutrirlo, educarlo y enseñarle a amar la razón, la ciencia y la humanidad en medio de un mundo completamente deshonesto. Este era su estado de ánimo cuando escribió su folleto De principatibus para lidiar con el mundo como realmente era, y no como debe ser.

Los constitucionalistas de hoy, y los conocedores de la Asociación Internacional de Derecho Constitucional, son como Maquiavelo. Nos hemos puesto nuestras túnicas curiales, hemos conversado con los antiguos y nos hemos recluido en nuestros estudios en San Andrea Percussina [ciudad italiana ubicada en Toscana]. La mayoría de nosotros no tenemos esperanza de poder político real o incluso capacidad de influencia. No tenemos voz en los consejos 
de los poderosos y el mundo que nos rodea es completamente corrupto. ¿Tendremos el coraje, aun así, de seguir el ejemplo de Maquiavelo? Él nos dio un valioso ejemplo en las dos grandes disciplinas de las cuales fue el fundador en la modernidad: constitucionalismo y ciencia política. Los Discorsi son una inestimable contribución a la primera de las anteriores y Il principe es una obra maestra de la ciencia política.

Maquiavelo en sus Discorsi examinó las mejores directrices de la política, las mejores estructuras de gobierno, del derecho, la libertad y el bien común, basados sobre todo en la experiencia, y particularmente la experiencia de Roma. En los Discorsi, Maquiavelo comprendió la constitución de la libertad en una república o un Estado libre. Miró en Il principe más al mundo en el cual él realmente vivió, y cómo un príncipe podría controlar y regular el Estado en un sistema de gobierno incapaz de ser republicano. El príncipe de Maquiavelo, ganando y manteniendo el poder en medio de la corrupción, tal vez pueda hacer algo bueno para el mundo, incluso podría encontrar la forma de constituir una república.

\section{Conclusiones}

Nicolás Maquiavelo fue el padre del constitucionalismo moderno. Este último comenzó con el estudio de las antiguas repúblicas y fue Maquiavelo quien inauguró esta nueva ciencia de la política. Quinientos años después de la composición de Il principe y los Discorsi todavía estamos trabajando en las implicaciones fruto de aplicar la razón a las leyes y a la constitución en busca de la justicia y del bien común. El constitucionalismo moderno y el republicanismo antiguo comparten tres creencias centrales. Primero, el gobierno debe servir a la justicia y al bien común. En segundo lugar, el gobierno debería alcanzar este objetivo a través de leyes conocidas y estables. Tercero, estas leyes estarán mejor aseguradas mediante los pesos y contrapesos de una constitución bien diseñada. Maquiavelo tomó la teoría y la experiencia de la Roma republicana y las aplicó a las dificultades políticas de su propia época. Esta aplicación de la razón a la gobernanza era nueva para los contemporáneos de Maquiavelo. Este fue el amanecer del nuevo mundo.

Maquiavelo fue el padre del constitucionalismo moderno, no solo en la importancia temporal de sus escritos para su época, sino también por su reconocida influencia en aquellos que lo siguieron. James Harrington, John Adams y el barón de Montesquieu, todos siguieron directamente a Maquiavelo en un intento por comprender mejor (en palabras de Adams): 
qué combinación de poderes en la sociedad, o qué forma de gobierno, permitirá la formación de leyes buenas y equitativas, una ejecución imparcial y una fiel interpretación de ellas, de modo que los ciudadanos puedan disfrutar constantemente de los beneficios de ellas y se asegure su continuidad (Adams 1787-1788, I.128).

Maquiavelo fue el "rastreador solitario"4 de la "política antigua" para el mundo moderno. Imperium legum potentiora quam hominum esto!

\section{Referencias}

Adams, J. (1787-1788). A Defence of the Constitutions of Government of the United States of America. London: Dilly.

Adams, J. (2000). Thoughts on Government. En J. Adams. The Revolutionary Writings of John Adams. Ed. C. B. Thompson. Indianapolis: Liberty Fund.

Aristoteles. (1957). Politics. (W. Ross, Trad.) Oxford: Oxford University Press.

Cicerón, M. (1913). De officiis. (W. Miller, Trad.) Cambridge: Harvard University Press.

Cicerón, M. (1928). De re publica et De legibus. (C. Keyes, Trad.) Cambridge: Harvard University Press.

Harrington, J. (1977). The Commonwealth of Oceana. En J. Harrington. The Political Works of James Harrington. Ed. J. G. A. Pocock. Cambridge: Cambridge University Press.

Livio, T. (1919). Ab urbe condita. Libri VI-X. (C. Walters y R. Conway, Trads) Oxford: Oxford University Press.

Livio, T. (1974). Ab urbe condita. Libri I-V. (R. Ogilvie, Trad.) Oxford: Oxford University Press.

Maquiavelo, N. 2001. El principe (4 ed.). (H. Puigdomenech, Trad.) Madrid: Tecnos.

Maquiavelo, N. 1987. Discursos sobre la primera década de Tito Livio. (A. Martínez Trad.) Madrid: Alianza.

4 N. del T. traduje la expresión "sole retriever" como "rastreador solitario". Retriever es un perro que rastrea las presas para el cazador. También puede ser traducido como "buscador solitario". 
Milton, J. (1991). Political Writings. Ed. M. Dzelzainis. Cambridge: Cambridge University Press.

Montesquieu, C. (1748). De l'esprit des lois. Geneva: Barillot.

Platón. (1902). Opera. Ed. I. Burnet. Oxford: Oxford University Press.

Plutarco. (1949-1971). Vitae. Ed. C. Lindskog and K. Ziegler. Leipzig: Teubner. Polybius. (1922). Historiae. (W. Paton, Trad.) Cambridge: Harvard University Press.

Rousseau, J. (1762). Du contrat social. Amsterdam: Marc Michel Rey.

Sallustius, G. (1931). Bellum catilinae et bellum iugurthinum. (J. Rolfe, Trad.) Cambridge: Harvard University Press.

Sellers, M. (1998). The Sacred Fire of Liberty: Republicanism, Liberalism, and the Law. Basingstoke: Macmillan.

Sellers, M. (2009). The Influence of Marcus Tullius Cicero on Modern Legal and Political Ideas. En Ciceroniana 13, pp. 245-280.

Sellers, M. (2014). What is the Rule of Law and Why Is It so Important? En The Legal Doctrines of the Rule of Law and the Legal State. Ed. R. Silkenat, J. Hickey y P. Barenboim. Dordrecht: Springer.

Sellers, M. (2004). Niccolò Machiavelli: Father of Modern Constitutionalism. En Ratio Juris, 28 (2), pp. 216-225.

Sidney, A. (1698). Discourses Concerning Government. London: Booksellers of London and Westminster.

Tácito, C. (1906). Annalium libri. Ed. C. Fisher. Oxford: Oxford University Press.

Tácito, C. (1911). Historiarum libri. Ed. C. Fisher. Oxford: Oxford University Press. 